\title{
A Thrust Balance for Low Power Hollow Cathode Thrusters
}

\author{
D.Frollani $^{a}$, M.Coletti ${ }^{\mathrm{b}}$ and S.B.Gabriel ${ }^{\mathrm{c}}$ \\ ${ }^{\mathrm{a}} \mathrm{PhD}$ student at Electronic and Computer Science, University of Southampton, df1r11@ soton.ac.uk \\ ${ }^{\mathrm{b} D i r e c t o r, ~ M a r s ~ S p a c e ~ L t d, ~ S O 14 ~ 5 F E ~ S o u t h a m p t o n, ~ m i c h e l e . c o l e t t i @ ~ m a r s-s p a c e . c o . u k ~}$ \\ ${ }^{a}$ Professor at Electronic and Computer Science, University of Southampton, sbg2@ soton.ac.uk
}

\begin{abstract}
A hanging thrust balance has been designed, manufactured and tested at the University of Southampton. The current design allows for direct steady thrust measurements ranging from $0.1 \mathrm{mN}$ to $3 \mathrm{mN}$ but this can be easily extended to measure thrust in a different range. Moreover the chosen balance design and the thrust measurement procedure allow for the cancellation of thermal drifts. The thrust balance was tested with a T6 hollow cathode thruster providing measurements with an uncertainty of about $9.7 \%$. The thrust data were compared to those obtained with another direct thrust balance, and they are in quantitative agreement being the maximum difference only $6 \%$.
\end{abstract}

Keywords. Direct measurements, thrust balance, electric propulsion, hollow cathode

\section{Introduction}

In recent years the trend in telecommunication satellites has been that of replacing chemical thrusters with electric propulsion (EP) to perform north/south station keeping (NSSK). Chemical propulsion $(\mathrm{CP})$ is currently still used to perform wheel momentum management and east/west station keeping (EWSK). The possibility of using EP to replace CP to perform EWSK and wheel momentum management is under investigation by many consortiums. In particular, Hollow Cathodes (HCs) have been investigated in [1] to give birth to an all-electric spacecraft since they can be highly integrated with the existing EP systems used for NSSK (hall thruster and gridded ion engines) reusing the existing tanks, fluid lines, flow control units and power supplies providing large dry mass savings. In the last decade HCs as standalone thrusters (Hollow Cathode Thrusters, HCTs) have been investigated at the University of Southampton (UoS), where the performances of QinetiQ T5 and T6 HCs for various discharge currents, mass flow rates and anode geometries [2, 3] have been measured with indirect thrust balances [4-7]. The use of an indirect thrust balance might not be completely reliable if the correction factor accounting for the influence of elastic or inelastic particle collisions with the target is not fully characterized and then used to correct the measurements. In particular, if all the collisions are elastic with particles reflected at $180^{\circ}$, the target might experience a force that is up to $100 \%$ higher than the real one hence producing measurement that significantly overestimates the real thrust value. Direct thrust measurements are performed typically using hanging $[8,9]$ and inverted or torsional pendulums $[10,11]$. In this article a new hanging pendulum balance is described: the present configuration was preferred to that of a null-reading balance because it allows for the using of a realtime acquisition method, which will be described in the paper, that, together with the selected balance design, allows for the cancellation of the thermal drifts. In the following paragraphs the experimental apparatus will be shown first, then the thrust balance will be described in detail and subsequently the 
thrust measurement technique, including the method of the cancellation of the thermal drifts, and the error budget will be discussed. Finally, the thrust measurements performed with a T6 HCT will be compared to those obtained with another direct thrust balance on the same device.

\section{Experimental Apparatus}

\subsection{The vacuum chamber}

The vacuum rig is shown in Figure 1 and consists of a stainless steel chamber $500 \mathrm{~mm}$ in diameter and $500 \mathrm{~mm}$ long. Vacuum is achieved with a water-cooled turbo molecular pump (Pfeiffer Balzers TPH 520KTG 500 1/s), controlled by a control unit made of a drive unit (Balzers TCP 380) and a controller (Balzers TCS 303) and backed by a rotary vane pump (Pfeiffer Balzers DUO 016B). Vacuum pressure is monitored via a pressure gauge package (Balzers TGP 30) consisting in a pirani gauge $\left(1000-5.4 \times 10^{-4}\right.$ mbar $)$ and a cold cathode gauge $\left(5 \times 10^{-3}-1 \times 10^{-9}\right.$ mbar $)$ placed on top of the chamber.

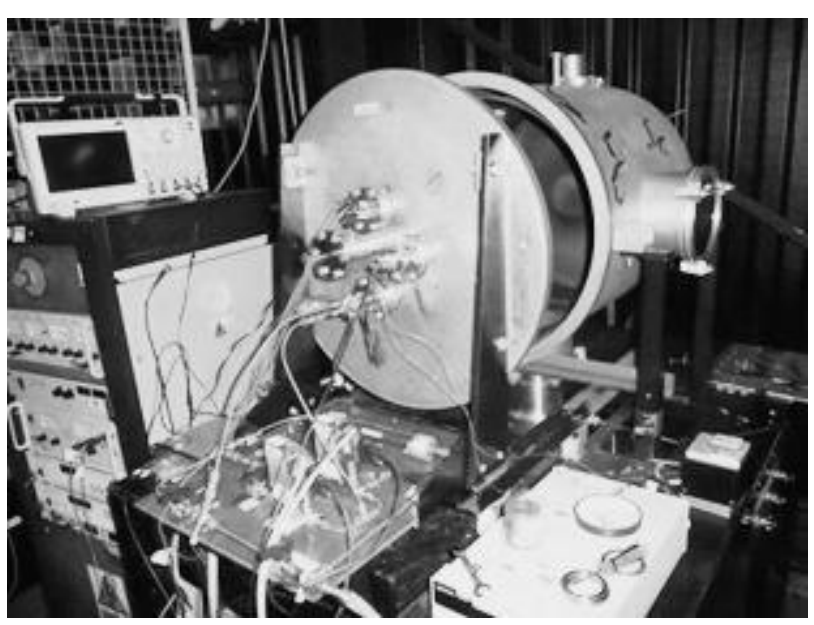

Figure 1. Vacuum chamber at the University of Southampton

Xenon gas grade 4.8 is supplied to the thruster from a cylinder which has a plug valve and a pressure reducer. To remove any oxygen impurity the gas is first driven towards an oxygen trap and then towards one of the two mass flow controllers and then into the vacuum chamber as shown in Figure 2. 


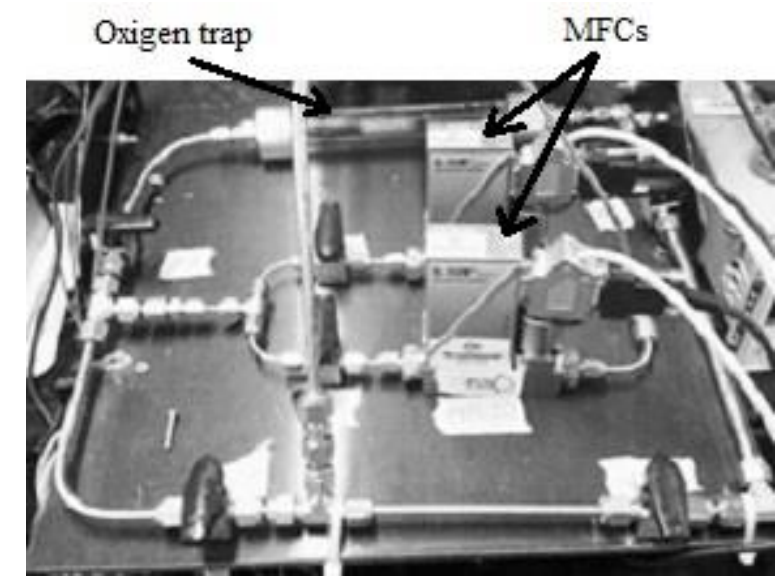

Figure 2. Fluidic line at the University of Southampton

The power supply units needed to run the thruster and the overall test setup are shown schematically in Figure 3:

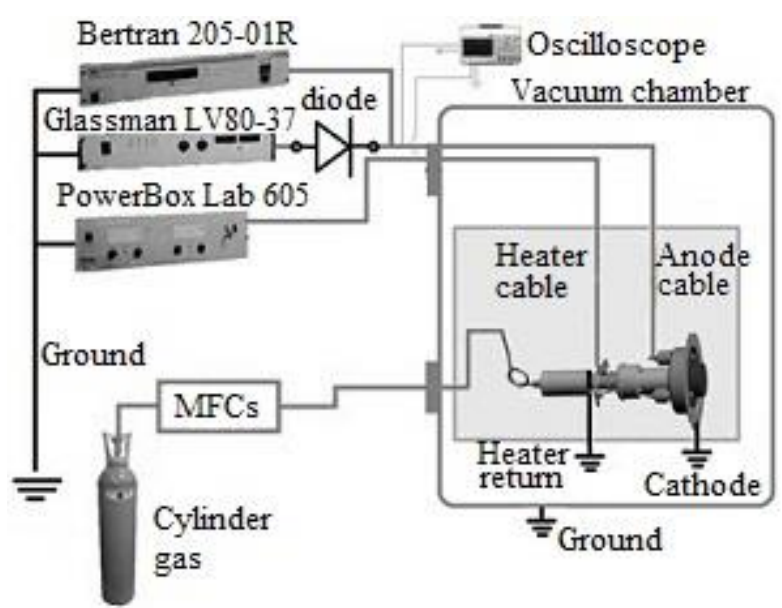

Figure 3. Test Setup

\subsection{The thrust balance}

The balance presented in this paper is a hanging pendulum balance and consists of a swinging arm fixed to a support structure via a Riverhawk 6012-800 flexural pivot [12] as shown in Figure 4 and Figure 5. 


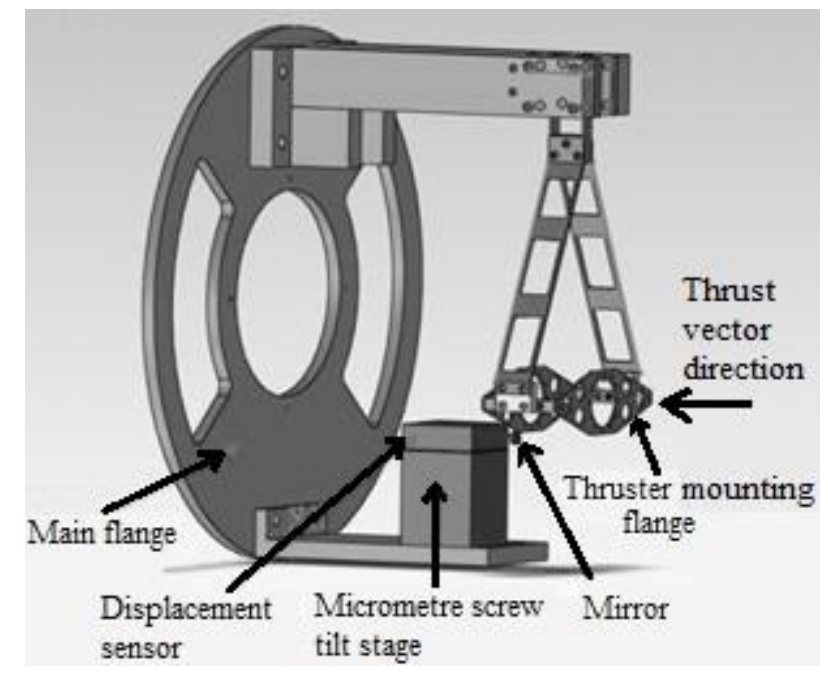

Figure 4. Complete thrust balance assembly schematic

The thruster is mounted at the end of the arm on the mounting flange and when thrust is applied the balance moves to a displaced equilibrium position. This equilibrium position is such that the torque generated by the thrust is balanced by the gravity and by the torsional spring constant of the flexural pivot. The displacement of the arm is measured using an optical sensor [13] placed on a tower where two micrometre screws and a tilt stage can be used for sensor alignment with a mirror connected to the swinging part of the balance

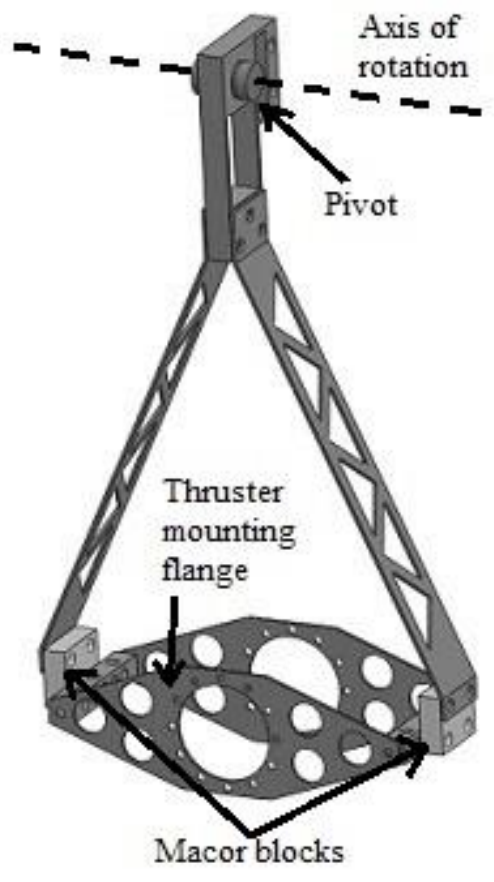

Figure 5. Swinging arm of the thrust balance 
As shown in Figure 6 the electrical and fluidic connections needed to operate the thruster are mechanically constrained to the swinging part of the balance via the flexural pivot clamp to minimize their effect on the balance dynamic response.
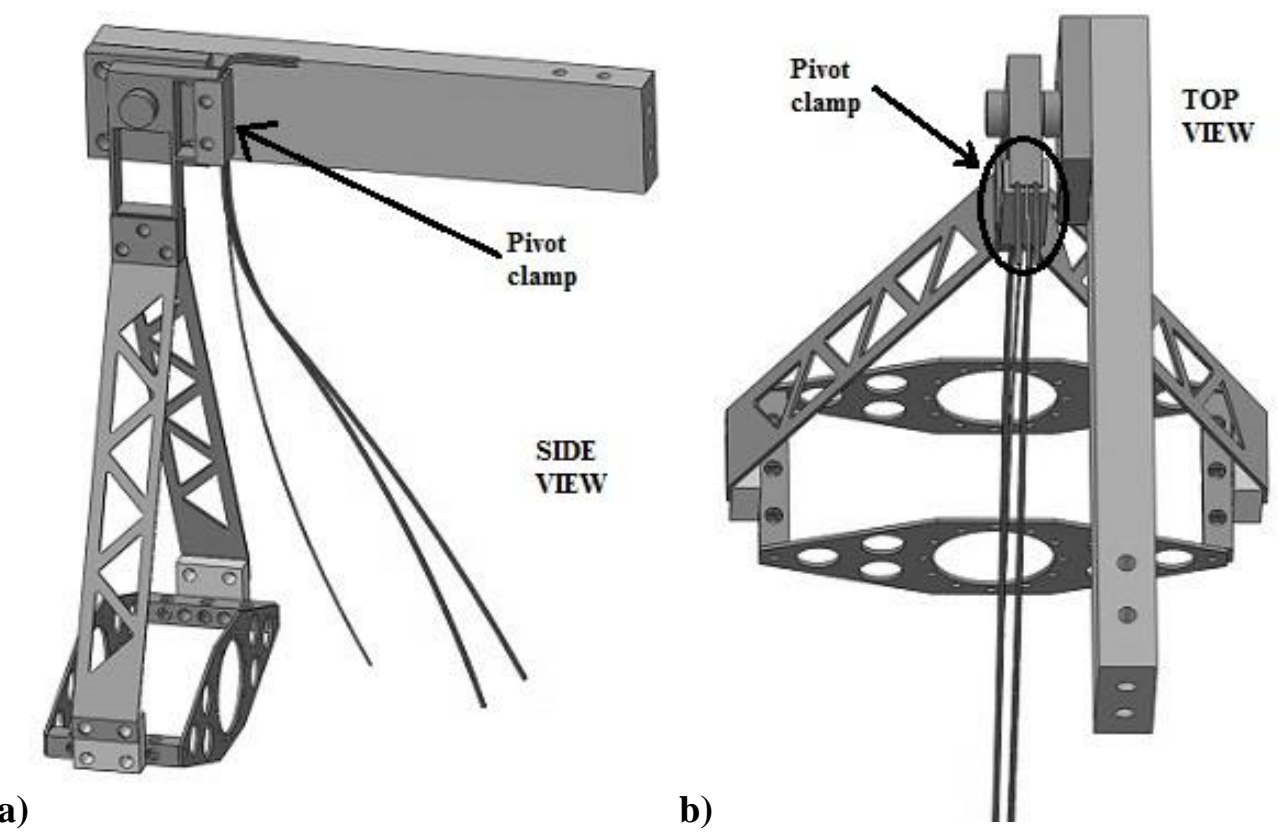

Figure 6. Electrical connections constrained to the thrust balance

\subsection{The displacement sensor}

The displacement sensor used is an interferometer (EUCLID) a complete and detailed description of which can be found in [13]. It is a 3R class laser with an operating wavelength of $667 \mathrm{~nm}$ and an optical power $<50 \mu \mathrm{W}$. The interferometer has to be aligned to the mirror on the swinging part of the thrust balance to obtain reliable measurements; the 'sweet-spot' $W_{D}$, i.e. the distance at which the tolerance to misalignment of the target mirror is maximised, is $6 \mathrm{~mm}$, and the working range $W_{R}$ is \pm 3 $\mathrm{mm}$ from the sweet-spot.

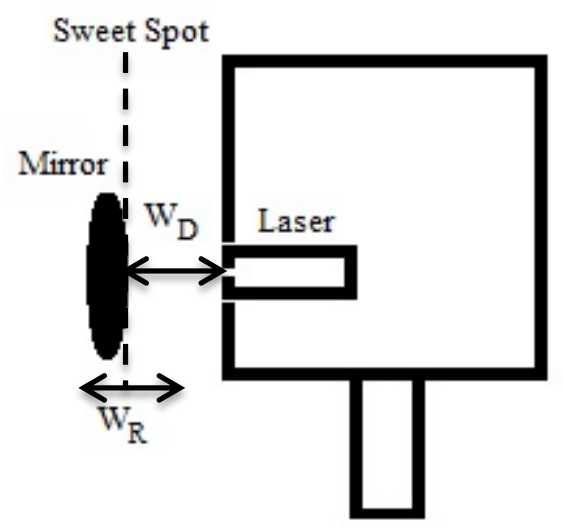

Figure 7. Working distance and sweet-spot of EUCLID 
According to EUCLID specifications the mirror reflectivity should be ideally $>0.9$ and its flatness at least $\lambda / 4$ : the one used at the University of Southampton is a Thorlabs PF05-03-F01 [14], which reflectivity and flatness are respectively $>0.9$ and $\lambda / 10$. The interferometer comes with an electric box and a Labview software which provides real-time tracking of the mirror, state of the alignment, and possibility of data logging.

\section{Thrust Balance Characterization}

\subsection{The working principle}

The thrust balance is schematically shown in Figure 8 , where $\vartheta$ and $\Delta x$ are respectively the angular and linear displacement, $T$ the thrust and $L_{t}$ the distance from the axis of rotation to the position where the thruster is mounted, $M$ and $L_{c m}$ the balance mass and the distance from the axis of rotation to the balance centre of mass, $g$ is the standard gravity and $L_{r}$ is the distance from the axis of rotation at which the mirror (i.e. the reading of the linear displacement) is placed.

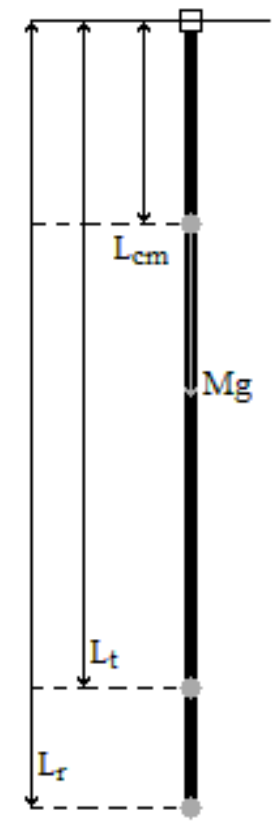

No thrust

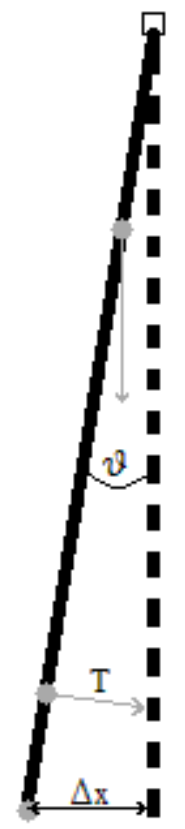

With thrust T

Figure 8. Thrust balance, schematics

Assuming that the balance angular displacement is small enough so that $\sin (\vartheta)=\vartheta$, the sum of the torques acting on the thrust balance, including the viscous friction term $\Gamma \dot{\vartheta}$, can be expressed as:

$$
J_{b a l} \ddot{\vartheta}+\Gamma \dot{\vartheta}+k \vartheta+M L_{c m} g \vartheta=T L_{t}
$$

where $J_{b a l}$ and $k$ are respectively the moment of inertia (with respect to the axis of rotation) and the torsional spring constant of the assembly formed by the pivot, the cables and propellant pipe. 
Equation (1) takes the shape of the classical pendulum equation when both the RHS and the LHS are divided by $J_{b a l}$ :

$$
\ddot{\vartheta}+2 \varsigma \omega_{n} \dot{\vartheta}+\omega_{n}^{2} \vartheta=\frac{T L_{t}}{J_{b a l}}
$$

In equation (2) $\zeta$ is the damping coefficient, expressed as:

$$
\varsigma=\frac{\Gamma}{2 J_{b a l} \omega_{n}}
$$

and $\omega_{n}$ is the natural angular frequency of the balance expressed as:

$$
\omega_{n}=\sqrt{\frac{k+M L_{c m} g}{J_{b a l}}}=\sqrt{\frac{K}{J_{b a l}}}
$$

With $K$ is indicated the total torsional spring constant of the whole balance assembly, including the gravity effect. For small angular displacement $(\sin (\vartheta)=\vartheta)$ it is possible to write:

$$
\vartheta=\frac{\Delta x}{L_{r}}
$$

Combining equations (2), (4) and (5) one can obtain an equation for the thrust in stationary conditions as:

$$
T=\frac{\omega_{n}{ }^{2} J_{b a l} \Delta x}{L_{t} L_{r}}
$$

According to equation (6), five parameters are needed to calculate the thrust. Two of them are geometric dimensions and were measured with a calliper and are $L_{l}=27.1 \pm 0.5 \mathrm{~cm}$ and $L_{r}=34.5 \pm 0.5$ $\mathrm{cm}$, which means an uncertainty of respectively $\pm 1.85 \%$ and $\pm 1.45 \%$. The other parameters needed are the linear displacement $\Delta x$, the natural angular frequency $\omega_{n}$ and the moment of inertia of the balance assembly $J_{b a l}$.

\subsubsection{Linear displacement evaluation}

The balance displacement is measured using the EUCLID interferometer described above; an example of an unfiltered sensor trace is reported in Figure 9 relative to the T6 running in discharge mode with xenon at $10 \mathrm{sccm}$ and $10 \mathrm{~A}$. When the thruster has reached a steady state, the value of the displacement can be obtained by turning off the discharge and the propellant feed at the same time. As soon as the thruster is switched off the balance will go back to its original equilibrium position hence an instantaneous measurement of the displacement is possible. Such displacement is obtained from 
the raw sensor data that are fitted before and after the thruster switch off with two straight lines, using which the displacement at the turn off time can be obtained as shown in Figure 9. It must be noted that the distance values when the thruster is on are negative since, given the geometrical arrangement of the balance, the swinging arm moves towards the sensor when a thrust is applied.

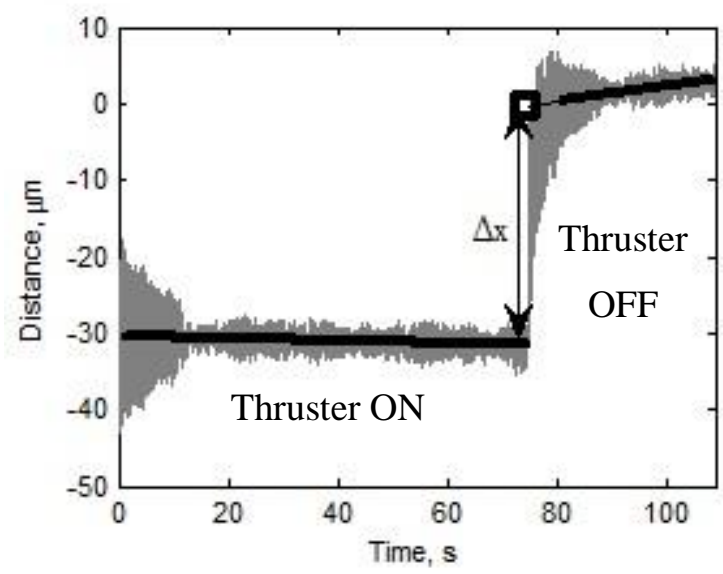

Figure 9. Displacement sensor trace for a T6 in discharge mode at $10 \mathrm{~A}$ and $10 \mathrm{sccm}$

The error on the displacement is mainly due to the balance oscillations around its equilibrium position. These oscillations are dependent on the thrust level at which the thruster is run and on the environment around the vacuum chamber. As an example, the analysis of the trace in Figure 9 shows an average linear displacement of $32.6 \mu \mathrm{m}$ with an uncertainty due to the balance oscillations around its equilibrium position of about $1.6 \mu \mathrm{m}$ resulting in an error of about $4.9 \%$.

\subsubsection{Natural angular frequency evaluation}

The natural angular frequency $\left(\omega_{n}=2 \pi f_{n}\right)$ of the balance assembly depends on the balance geometry, on the position of the thruster under test and on the cables connected to it as shown in Figure 6. The natural frequency $f_{n}$ can be calculated via a Fourier Transform of the acquired displacement signal. In Figure 10 an example evaluation of the natural frequency is shown.

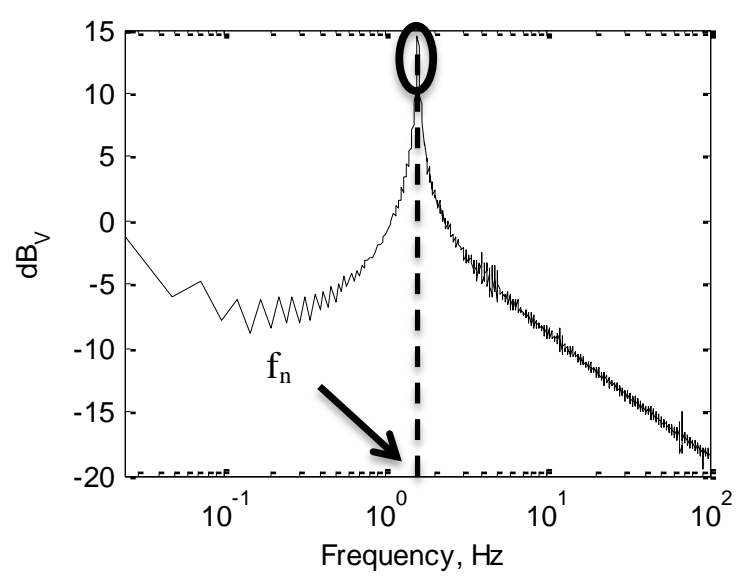


Figure 10. Example of Fourier Transform to evaluate the natural frequency

As reported in [15], the uncertainty in the evaluated natural angular frequency is given by:

$$
\delta \omega_{n}=2 \pi f_{s} \frac{1}{2(P / 2+1)}
$$

where $f s$ is the signal sampling frequency and $P$ is the power of 2 closest to the number of acquired samples. Since the signal was acquired for at least 5 minutes every time using an oscilloscope with a sampling frequency of $25 \mathrm{kHz}$, the uncertainty in the natural angular frequency has an order of magnitude of $1 \mathrm{mHz}$. Since the natural angular frequency has an order of magnitude of $1 \mathrm{~Hz}$, its uncertainty is of the order of $0.1 \%$ hence can be neglected.

\subsubsection{Moment of inertia evaluation}

The moment of inertia of the thrust balance assembly $J_{b a l}$ can be obtained with a dynamic calibration of the system. In particular such calibration can be carried out with a procedure consisting in applying different known weights to the balance. In fact, if an extra weight is attached to each side of the balance at a distance $L_{m}$ from the centre of rotation, the balance new natural angular frequency can be written following the procedure carried out in equation (4) as:

$$
\omega_{n}=\sqrt{\frac{K+2 m L_{m} g}{J_{b a l}+2 J_{m}}}
$$

where $J_{m}$ is the moment of inertia of the weight relative to the balance rotational axis. Equation (8) can be re-arranged as:

$$
2 \omega^{2} J_{m}-2 m L_{m} g=-J_{b a l} \omega_{n}^{2}+K
$$

If the extra weights have a known mass and have a simple shape the LHS of equation (9) can be easily obtained measuring the natural angular frequency of the balance $\left(\omega_{n}\right)$, measuring where the weights are positioned (distance from the balance axis of rotation $L_{m}$ ) and calculating their moment of inertia $\left(J_{m}\right)$. By attaching different weights and obtaining different values for the LHS of equation (9) it is possible to linearly fit the data to obtain the balance total torsional spring constant and total moment of inertia. In particular the absolute value of the slope of the linear fit will be $J_{b a l}$, whereas the intercept is $K$. 

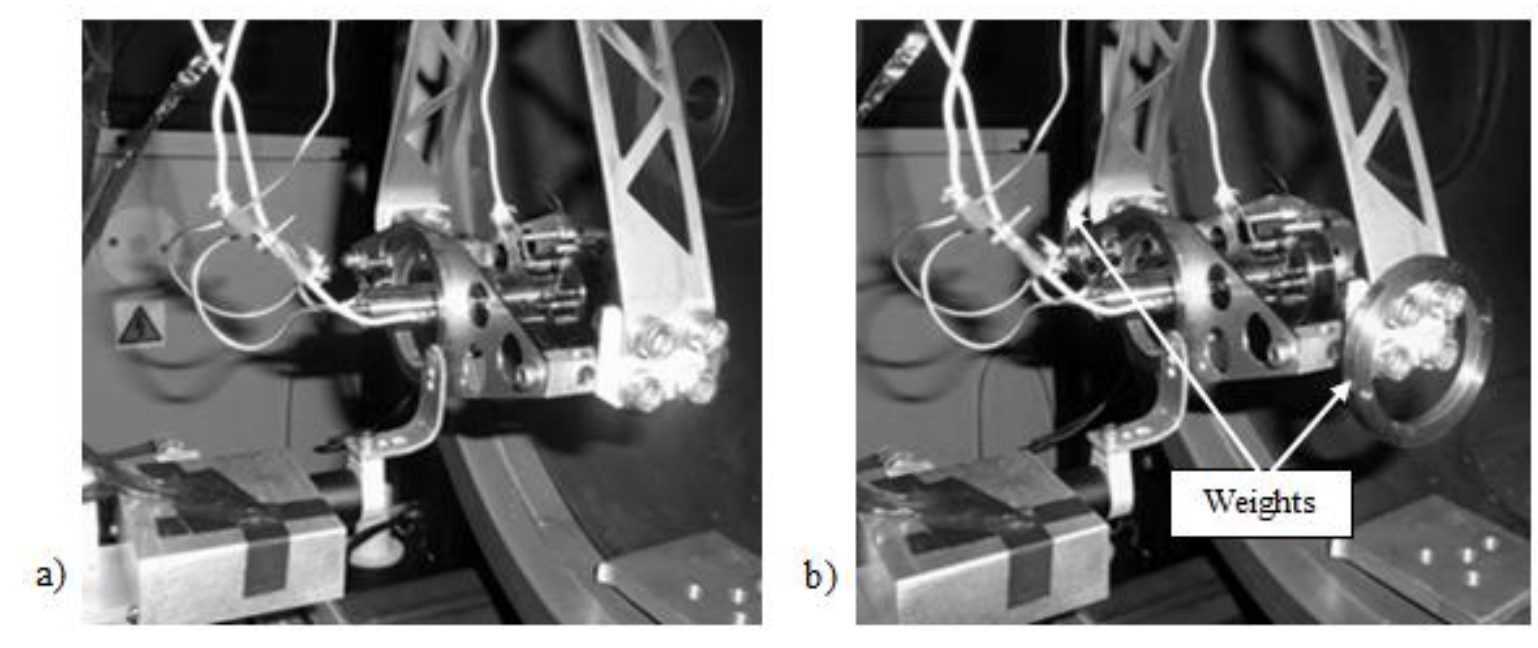

Figure 11. Thrust balance a) without and b) with weights

Two pairs of different annular weights have been manufactured and their geometrical parameters are shown in Table 1:

Table 1. Geometrical parameters of the weights

\begin{tabular}{ccc}
\hline Weight & 'Small' & 'Big' \\
\hline$D_{\text {ext }}, \mathrm{mm}$ & 54 & 54 \\
$D_{\text {int }}, \mathrm{mm}$ & 42 & 42 \\
$t h, \mathrm{~mm}$ & 5 & 15 \\
$m, \mathrm{~g}$ & 14.2 & 35.6 \\
$L_{m}, \mathrm{~mm}$ & 282 & 282 \\
\hline
\end{tabular}

In Table 2 the different configurations of weights used for this test are reported:

Table 2. Configuration of weights used

\begin{tabular}{ccc}
\hline \multirow{2}{*}{ Config. no. } & \multicolumn{2}{c}{ Weights used } \\
& Left side & Right side \\
\hline $\mathbf{1}$ & - & - \\
$\mathbf{2}$ & Small & Small \\
$\mathbf{3}$ & Big & Big \\
$\mathbf{4}$ & Big+Small & Big+Small \\
\hline
\end{tabular}

Figure 12 shows the result of the dynamic calibration, where the four configurations were fitted with a straight line as per equation (9). Fifteen tests were carried out for each configuration but for clarity in Figure 12 only the mean value of each configuration is shown. The standard deviation of the mean value is $<<1 \%$ hence negligible and not reported in Figure 12. 


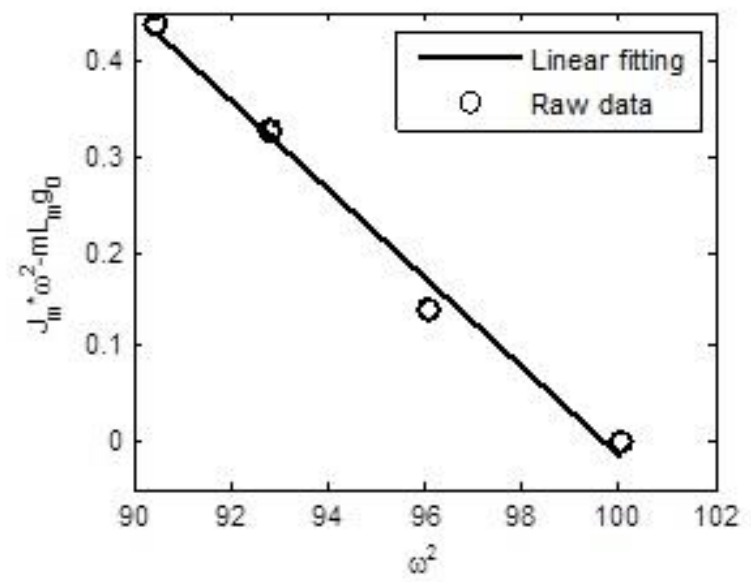

Figure 12. Dynamic Calibration for the calculation of the moment of inertia for the T6 balance assembly

The moment of inertia for this configuration is $J_{b a l}=0.0466 \mathrm{~kg} \mathrm{~m}^{2}$. The uncertainty of the moment of inertia was calculated as that of the fitting, in agreement with what reported in [16], and was found to be $\pm 0.0037 \mathrm{~kg} \mathrm{~m}^{2}$ hence about $8 \%$ of the nominal $J_{b a l}$ value.

\subsection{Evaluation of thermal effects on the measurements}

The calculation of thrust may be affected by thermal drifts due to the heat produced by inefficiencies of the thruster. In Figure 9, for example, the sensor trace shows that the mean value of the displacement $\Delta x$ increases after the switch-off due to thermal effects. In particular, the thermal effects may affect the geometrical parameters as well as the balance moment of inertia and natural angular frequency needed to calculate the thrust as shown in equation (6). Considering that the balance is made of stainless steel (that has a thermal expansion coefficient of $17 \times 10^{-6} \mathrm{~K}^{-1}$ [17]) a balance temperature increase of $500{ }^{\circ} \mathrm{C}$, which is a very pessimistic hypothesis, will cause an additional error due to thermal expansion of about $0.7 \%$ on $L_{t}$ and $L_{r}$. Considering that the moment of inertia is proportional to the balance mass and to the square of the balance dimension the error on $J_{b a l}$

will be twice that on $L_{t}$ and $L_{r}\left(J_{b a l} \sim m L^{2} \mapsto \delta J_{b a l} / J_{b a l} \sim 2 \delta L / L\right)$ [16] hence about $1.4 \%$. A temperature increase will also affect the mechanical properties of the pivot hence having an influence on the spring constant $k$, ultimately modifying the natural angular frequency of the balance. To reduce the heat conduction towards the pivot, blocks of Macor (which has a thermal conductivity of $1.46 \mathrm{~W}$ $\mathrm{m}^{-1} \mathrm{~K}^{-1}[18]$ ) are used as shown in Figure 5 to connect the thruster mounting balance to the swinging balance arm. Nevertheless, considering that the value of $\omega_{n}$ used in equation (6) is calculated from the displacement sensor trace (hence the knowledge of the value of $k$ and/or of its trend with temperature is not needed) and that the evaluation of the displacement is done instantaneously at the turn off as described above in paragraph 3.1.1, it can be seen how the thermal effects on $\Delta x$ and $\omega_{n}$ are automatically taken into account by the thrust measurement procedure and by the use of equation (6). 
Considering all this it can be seen how no errors relative to $\Delta x$ and $\omega_{n}$ should be included due to thermal effects.

\subsection{The error budget}

The experimental mean value of the thrust is obtained using equation (6). Its value is affected by an uncertainty which will be calculated within this paragraph. It is useful to remember that in equation (6) $L_{t}$ and $L_{r}$ have been measured with a calliper, $J_{b a l}$ is obtained with the dynamic calibration, and $\Delta x$ and $\omega_{n}$ are obtained from the displacement sensor trace. The error on the thrust can be expressed as reported in [16]:

$$
\delta T=T \sqrt{\left(\frac{\delta \Delta x}{\Delta x}\right)^{2}+\left(2 \frac{\delta \omega_{n}}{\omega_{n}}\right)^{2}+\left(\frac{\delta L_{t}}{L_{t}}\right)^{2}+\left(\frac{\delta L_{r}}{L_{r}}\right)^{2}+\left(\frac{\delta J_{b a l}}{J_{b a l}}\right)^{2}}
$$

Considering all what has been reported in the previous paragraphs, a preliminary balance error budget is reported in Table 3:

Table 3. Error budget

\begin{tabular}{cccc}
\hline Quantity & Error & $\begin{array}{c}\text { Thermal effects } \\
\text { at 500 }\end{array}$ & $\begin{array}{c}\text { Total error at } \\
\mathbf{5 0 0}{ }^{\circ} \mathrm{C}\end{array}$ \\
\hline $\boldsymbol{L}_{\boldsymbol{t}}$ & $\pm 1.85 \%$ & $\pm 0.7 \%$ & $\pm 1.98 \%$ \\
$\boldsymbol{L}_{\boldsymbol{r}}$ & $\pm 1.45 \%$ & $\pm 0.7 \%$ & $\pm 1.61 \%$ \\
$\boldsymbol{J}_{\boldsymbol{b a l}}$ & $\pm 8.0 \%$ & $\pm 1.4 \%$ & $\pm 8.1 \%$ \\
$\boldsymbol{\omega}_{\boldsymbol{n}}$ & $<<1 \%$ & $0 \%$ & $<<1 \%$ \\
& & & Measurement \\
$\boldsymbol{\Delta} \boldsymbol{x}$ & Measurement & $0 \%$ & dependent $\sim 5 \%$ \\
\hline Thrust & $\sim \pm 9.7 \%$ & $\pm 1.7 \%$ & $\sim \pm 9.9 \%$ \\
\hline
\end{tabular}

It should be noted that the last three terms in equation (10) are inclusive of the error due to the thermal effects, as shown in equation (11) in an example for $L_{t}$ :

$$
\left(\frac{\delta L_{t}}{L_{t}}\right)^{2}=\left(\frac{\delta L_{t}}{L_{t}}\right)_{\text {nothermal }}^{2}+\left(\frac{\delta L_{t}}{L_{t}}\right)_{\text {thermal }}^{2}
$$

\section{Comparison with another thrust balance}

The direct thrust measurements carried out at the UoS on the T6 HCT were compared to those obtained at Aerospazio Tecnologie s.r.l (AER) on the same device. 


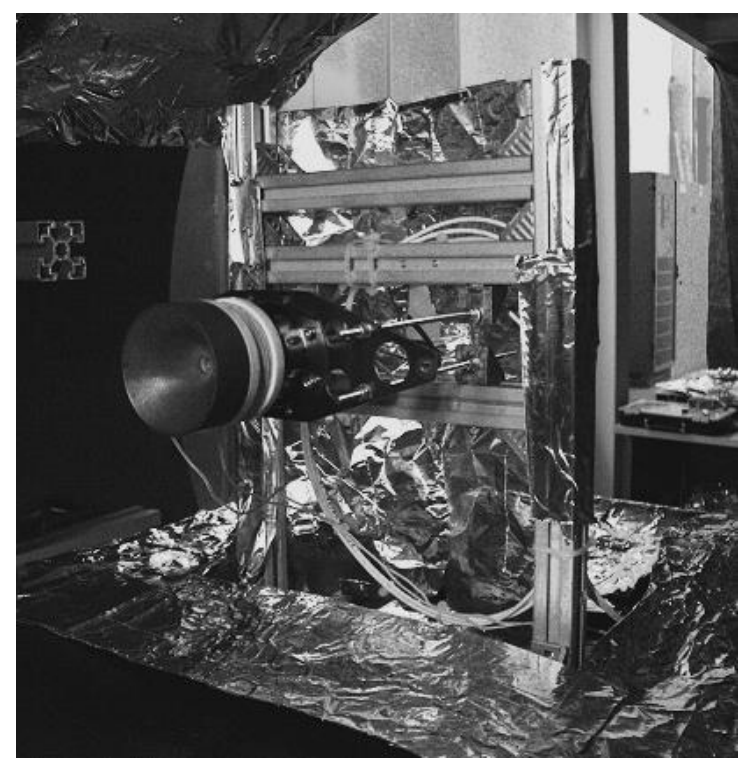

Figure 13. The T6 HCT on the thrust balance at AER

The thrust balance at AER is a direct one and consists of a parallelogram composed of two plates (an upper one and a lower one) connected together by means of four bars and the corresponding hinges. The lower plate is fixed and connected to the test facility whereas the thruster can be accommodated on the upper movable part. This balance works on the principle of the null reading and has been already successfully used to characterize the performance of other electric propulsion devices.
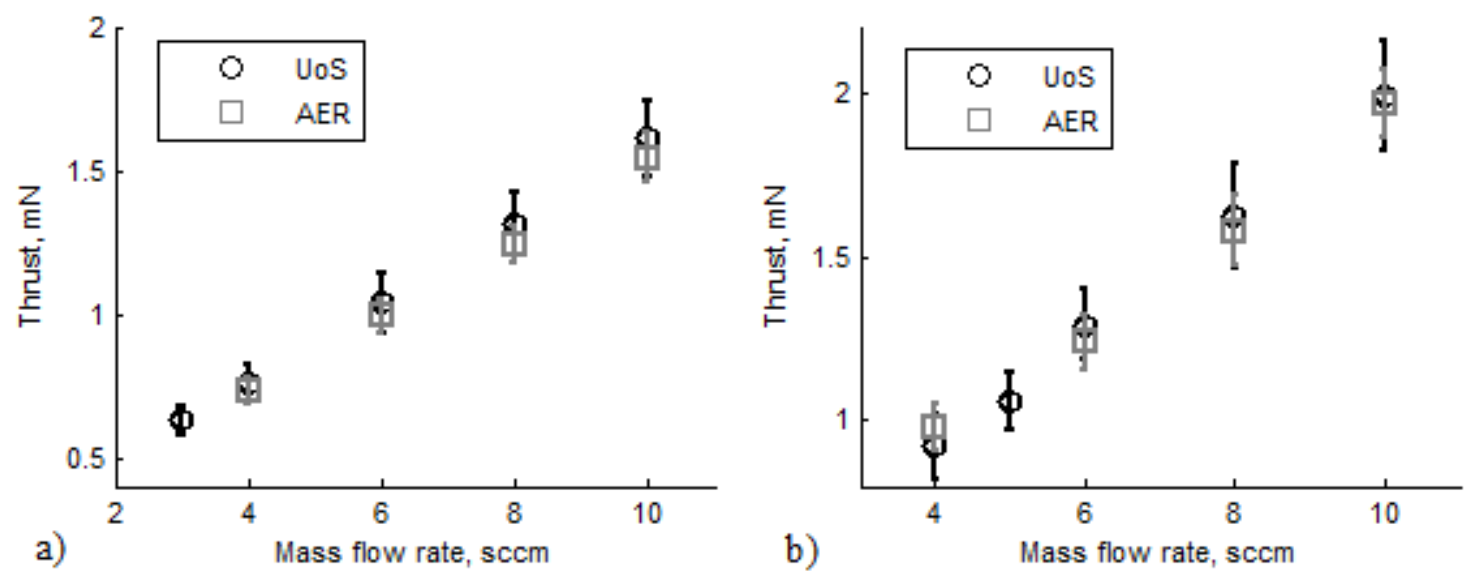

Figure 14. Direct thrust measurement on the T6 HCT at AER and UoS at a) $10 \mathrm{~A}$ and b) $15 \mathrm{~A}$

It should be noted that at the UoS the temperature of the thrust balance was monitored via a PT1000 RTD temperature sensor positioned on the fixed part of the balance near the pivot and the maximum change in temperature during the tests was under $40{ }^{\circ} \mathrm{C}$. Considering that the uncertainty introduced by thermal effect scale linearly with the temperature change and comparing the measured temperature increase with that used in Table 3, it can be concluded how the uncertainty relative to thermal effect in this case is negligible $(<0.1 \%)$. The thrust data obtained with the two different direct thrust balances are in good quantitative agreement being the maximum difference only $6 \%$ at $4 \mathrm{sccm}$ and $15 \mathrm{~A}$. 


\section{Conclusions}

A direct thrust balance designed, manufactured and tested at the University of Southampton in collaboration with Mars Space Ltd has been presented. The development of such a device is fundamental for low cost and easy access HCTs characterization and performance assessment. The presented configuration, allowing for direct steady thrust measurement, is a hanging pendulum where a mirror attached to the swinging arm is tracked by an interferometer which gives the real-time displacement. Real-time acquisition of the linear displacement and measurement of the natural angular frequency, which are used to calculate the thrust, allow for the cancellation of the thermal drift and the uncertainties due to it. The balance was found to have a total uncertainty on the thrust measurement of about $9.7 \%$ in its current configuration. The thrust results obtained on a T6 HCT were compared to those obtained on the same thruster with another direct thrust balance, designed and manufactured at Aerospazio Tecnologie, in Italy. The results from the two thrust balances are in good quantitative agreement being the maximum difference only $6 \%$ and this will give us the possibility to perform future measurements confidently.

\section{A. Appendix - Nomenclature}

\begin{tabular}{|c|c|c|c|c|c|}
\hline AER & $=$ & Aerospazio Tecnologie s.r.1. & $\mathrm{L}_{\mathrm{m}}$ & $=$ & Mass application distance \\
\hline $\mathrm{CP}$ & $=$ & Chemical propulsion & $\mathrm{L}_{\mathrm{r}}$ & $=$ & Distance to mirror tracking \\
\hline$D_{\text {ext }}$ & $=$ & External diameter & $\mathrm{L}_{\mathrm{t}}$ & $=$ & Distance to thrust application \\
\hline $\mathrm{D}_{\mathrm{int}}$ & $=$ & Internal diameter & M & $=$ & Mass of the balance \\
\hline EP & $=$ & Electric propulsion & $\mathrm{m}$ & $=$ & Mass of the weights \\
\hline EWKS & $=$ & East West station keeping & MFC & $=$ & Mass flow controller \\
\hline$f_{n}$ & $=$ & Natural frequency & NSSK & $=$ & North South station keeping \\
\hline$f_{s}$ & $=$ & Sampling frequency & $\mathrm{T}$ & $=$ & Thrust \\
\hline $\mathrm{g}$ & $=$ & Standard gravity & th & $=$ & Thickness \\
\hline $\mathrm{HC}$ & $=$ & Hollow cathode & UoS & $=$ & University of Southampton \\
\hline $\mathrm{HCT}$ & $=$ & Hollow cathode thruster & $\mathrm{W}_{\mathrm{D}}$ & $=$ & EUCLID’s sweet-spot \\
\hline $\mathbf{J}_{\text {bal }}$ & $=$ & Balance moment of inertia & $\mathrm{W}_{\mathrm{R}}$ & $=$ & EUCLID's working range \\
\hline $\mathbf{J}_{\mathrm{m}}$ & $=$ & Weight moment of inertia & $\omega_{\mathrm{n}}$ & $=$ & Natural angular frequency \\
\hline K & $=$ & Total spring constant & $\vartheta$ & $=$ & Angular displacement \\
\hline $\mathrm{k}$ & $=$ & Pivot spring constant & & & \\
\hline $\mathrm{L}_{\mathrm{cm}}$ & $=$ & Centre of mass distance & & & \\
\hline
\end{tabular}

\section{REFERENCES}

1. Coletti, M., et al., European Student Moon Orbiter Solar Electric Propulsion Subsystem Architecture - An All-Electric Spacecraft, in International Electric Propulsion Conference. 2007. p. IEPC-2007- 111.

2. Gessini, P., Cathode Thrust Measurements Using a Target. 2007, University of Southampton. 
3. Grubisic, A.N., Microthrusters Based on the T5 and T6 Hollow Cathodes. 2009, University of Southampton.

4. Gessini, P. and S.B. Gabriel, A Study of the Thrust Generated by a T6 Hollow Cathode, in 42nd AIAA/ASME/SAE/ASEE Joint Propulsion Conference \& Exhibit. 2006. p. AIAA 20065265 .

5. Gessini, P., S.B. Gabriel, and D.G. Fearn, Hollow Cathode Thrust Measurement Using a Target: System Calibration and Development, in 38th AIAA/ASME/SAE/ASEE Joint Propulsion Conference \& Exhibition. 2002. p. AIAA 2002-4104.

6. Grubisic, A.N. and S.B. Gabriel, Development of an indirect counterbalanced pendulum optical-lever thrust balance for micro- to millinewton thrust measurement. Measurement Science \& Technology, 2010. 21(10).

7. Grubisic, A.N., S.B. Gabriel, and D.G. Fearn, Preliminary Thrust Characterization of the TSeries Hollow Cathodes for All-Electric Spacecraft, in 30th International Electric Propulsion Conference. 2007. p. IEPC-2007-81.

8. Packan, D., J. Bonnet, and S. Rocca, Thrust measurement with the ONERA micronewton balance, in 30th International Electric Propulsion Conference. 2007.

9. Diamant, K.D., et al., Thrust Stand Characterization of the NASA Evolutionary Xenon Thruster. Journal of Propulsion and Power, 2011. 27(4): p. 777-785.

10. Koizumi, H., K. Komurasaki, and Y. Arakawa, Development of thrust stand for low impulse measurement from microthrusters. Review of Scientific Instruments, 2004. 75(10): p. 31853190.

11. Ziemer, J.K., Performance Measurements Using a Sub-Micronewton Resolution Thrust Stand, in International Electric Propulsion Conference. 2001. p. IEPC-01-238.

12. RiverHawk Flexural Pivots, Double-Ended Pivot Part No. 6012-800. Datasheet available at the time of publication at $\mathrm{http}: / / \mathrm{www}$. flexpivots.com/doubleendedpivotbearings.php.

13. Aston, S.M., Optical Read-Out Techniques for the Control of Test-Masses in Gravitational Wave Observatories. 2011, University of Birmingham.

14. Thorlabs, Mirror Part no. PF05-03-F01. Datasheet available at the time of publication at http://www.thorlabs.de/catalogpages/V21/774.PDF.

15. Oppenheim, A.V. and R.W. Schafer, Discrete-time signal processing. 2009: Prentice Hall.

16. Taylor, J.R., An introduction to error analysis. 1996: UniversityScienceBooks.

17. Kovach, C.W., High Performance Stainless Steel. Nickel Institute.

18. Rutt, H., Technical Note 02-053: The thermal conductivity of macor at intermediate cryogenic temperatures. 2002, Thomas Jefferson National Accelerator Facility. 